\title{
Editorial
}

\section{Theory and Applications of Periodic Solutions and Almost Periodic Solutions}

\author{
Yonghui Xia \\ Department of Mathematics, Zhejiang Normal University, Jinhua 321004, China \\ Correspondence should be addressed to Yonghui Xia; yhxia@zjnu.cn
}

Received 17 September 2013; Accepted 17 September 2013

Copyright (C) 2013 Yonghui Xia. This is an open access article distributed under the Creative Commons Attribution License, which permits unrestricted use, distribution, and reproduction in any medium, provided the original work is properly cited.

Theory of periodic solutions and almost periodic solutions is an important and well-established branch of the modern theory of differential equations concerned, in a broad sense, with the study of periodic (almost periodic) phenomena arising in applied problems in technology, natural, and social sciences. For example, to explore the impact of environmental factors in mathematical biology, the assumption of periodicity of parameters is more realistic and important due to many periodic factors such as seasonal effects of weather, food supplies, mating habits, and harvesting. However, if the various constituent components of the temporally nonuniform environment are with incommensurable (nonintegral multiples) periods, then one has to consider the environment to be almost periodic. It is well known that existence and nonexistence of periodic (or almost periodic) solutions to a given system are the classical theory of oscillation theory, which is an intrinsic feature of many dynamical systems.

This special issue places its emphasis on the study of periodic or almost periodic solutions of initial/boundary value problems for ordinary differential equations. It includes many topics such as almost automorphic mild solutions, biological systems, neural networks, oscillation criteria, delay differential equation, stochastically epidemic model, initial value problems, Lyapunov function, complex dynamical behaviors, stability and bifurcation, and impulsive differential equation.

All manuscript submitted to this special issue went through a thorough peer-refereeing process. Based on the reviewers' reports, we collect 16 original research articles by more than fifty active international researchers on differential equations.
To be a more comprehensive description for all articles in this special issue, we provide a short editorial note summering each paper.

$\mathrm{Z}$. Hu and Z. Jin establish new existence and uniqueness theorems for almost automorphic mild solutions to neutral parabolic nonautonomous evolution equations with nondense domain. A unified framework is set up to investigate the existence and uniqueness of almost automorphic mild solutions to some classes of parabolic partial differential equations and neutral functional differential equations.

S. Xie et al. studied almost periodic solutions for WilsonCowan type model with time-varying delays. Some Su cient conditions for the existence and delay-based exponential stability of a unique almost periodic solution are established.

$\mathrm{S}$. Li showed that the system considered has more complicated dynamics, including (1) high-order quasi-periodic and periodic oscillation, (2) period-doubling and halving bifurcation, (3) nonunique dynamics (meaning that several attractors coexist), and (4) chaos and attractor crisis.

$\mathrm{H}$. Wu et al. studied a Nicholson's blowflies model with feedback control and time delay. By applying the comparison theorem of the differential equation and fluctuation lemma and constructing a suitable Lyapunov functional, sufficient conditions which guarantee the permanence, extinction, and existence of a unique globally attractive positive almost periodic solution of the system are obtained.

W. Wang et al. presented a theoretical analysis of processes of pattern formation that involves organisms distribution and their interaction of spatially distributed population with self- as well as cross-diffusion in a Holling-Tanner predator-prey model; the sufficient conditions for the Turing 
instability with zero-flux boundary conditions are obtained; Hopf and Turing bifurcation in a spatial domain is presented, too.

T. Zhang investigated a stochastic epidemic model with time delays. By using Lyapunov functionals, he obtained stability conditions for the stochastic stability of endemic equilibrium.

K.-C. Wang concerned with the Dullin-Gottwald-Holm (DGH) equation with strong dissipation. He established a sufficient condition to guarantee global in time solutions.

Z. Yue et al. investigated the dynamics of a diffusive ratio-dependent Holling-Tanner predator-prey model with Smith growth subject to zero-flux boundary condition. Some qualitative properties, including the dissipation, persistence, and local and global stability of positive constant solution are discussed.

B. Yang investigated the spatiotemporal dynamics of a diffusive ratio-dependent Holling-Tanner predator-prey model with Smith growth subject to zero-flux boundary condition.

$\mathrm{H}$. Ma et al. constructed a new Lyapunov function for a class of predation models.

J. Chen studied the atom-bond connectivity index of catacondensed polyomino graphs.

P. Tang quantitatively studied the effect of delay on selection dynamics in long-term sphere culture of cancer stem cells (CSCs); a selection dynamic model with time delay is proposed.

H. Chen et al. derived several sufficient conditions for monotonicity of eventually positive solutions on a class of second order perturbed nonlinear difference equation.

$\mathrm{X}$. Liu et al. considered a predator prey system with Holling III functional response and constant prey refuge. By using the Dulac criterion, we discuss the global stability of the positive equilibrium of the system. By transforming the system to a Lienard system, the conditions for the existence of exactly one limit cycle for the system are given.

Lian et al. presented a theoretical analysis of evolutionary process that involves organisms distribution and their interaction of spatial distribution of the species with self and crossdiffusion in a Holling III ratio-dependent predator-prey model.

$\mathrm{X}$. Wang et al. are concerned with the reaction-diffusion Holling-Tanner prey-predator model considering the Allee effect on predator, under zero-flux boundary conditions.

\section{Acknowledgment}

We would also like to thank the editorial board members of this journal, for their support and help throughout the preparation of this special issue. 


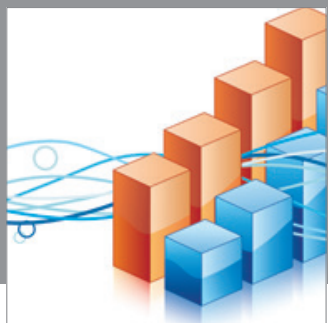

Advances in

Operations Research

mansans

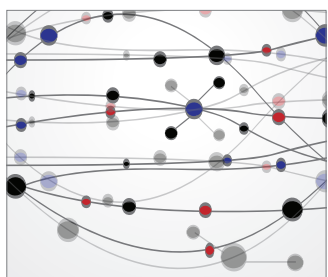

The Scientific World Journal
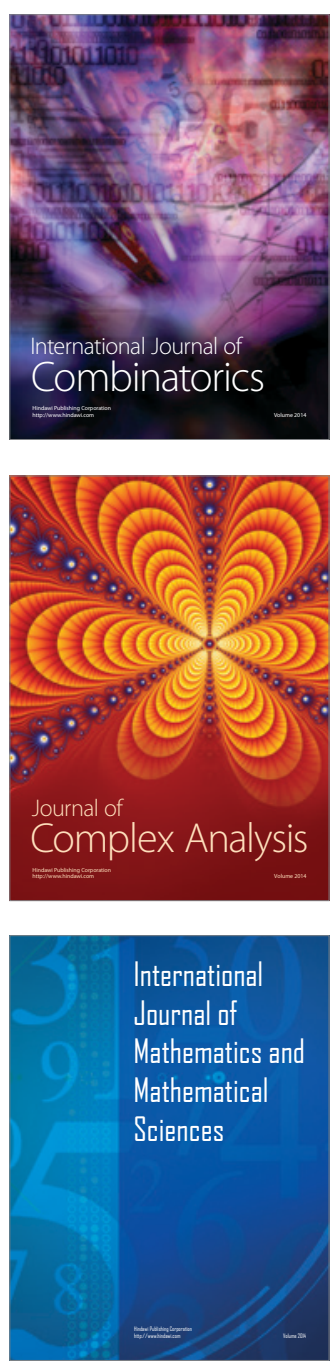
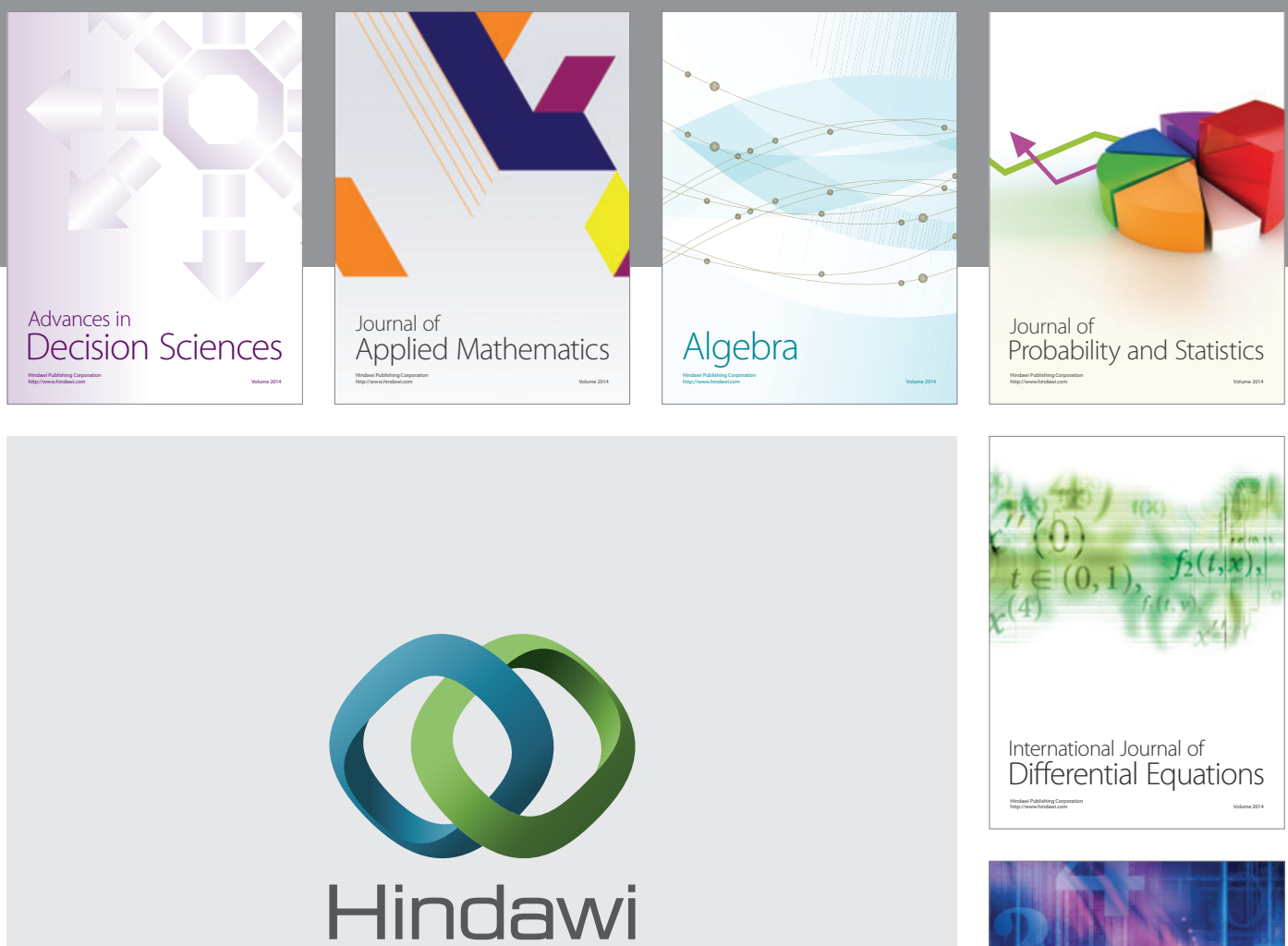

Submit your manuscripts at http://www.hindawi.com
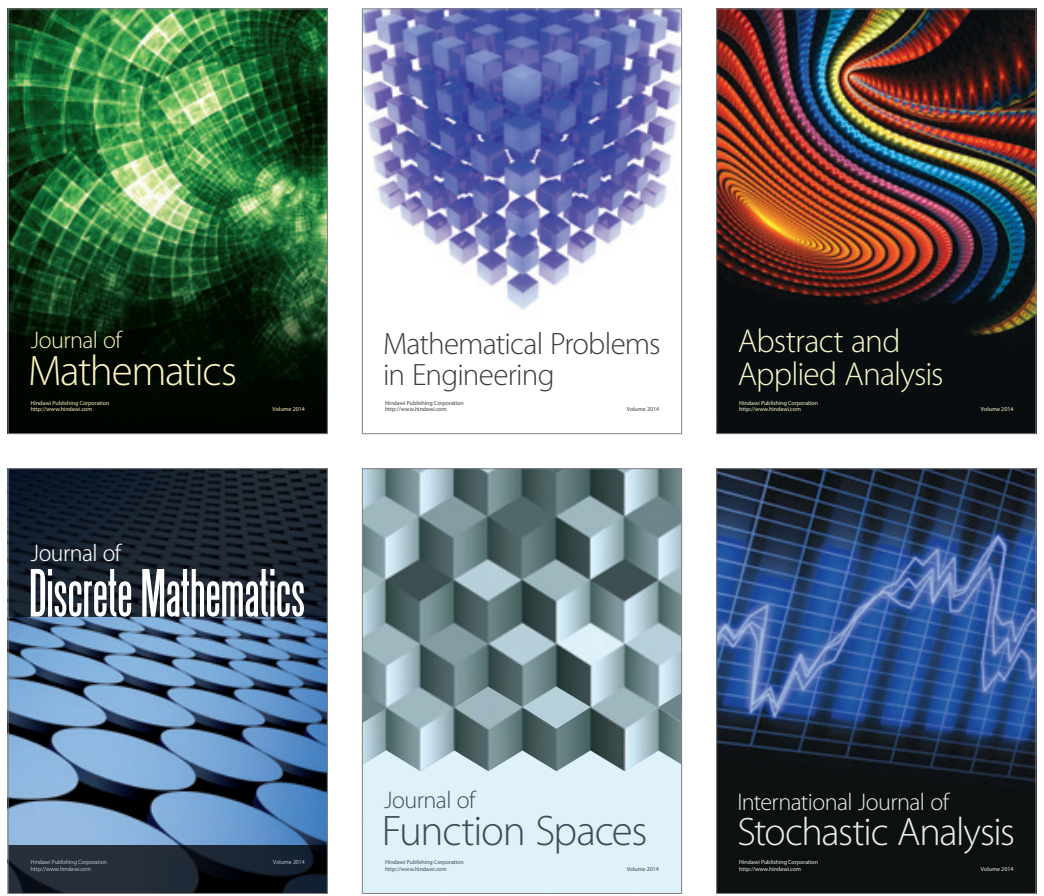

Journal of

Function Spaces

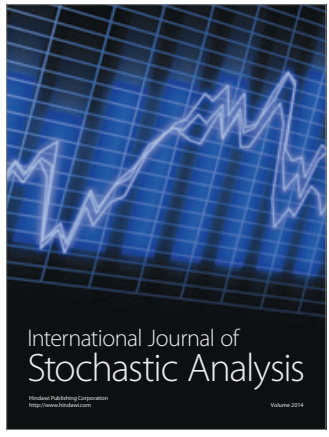

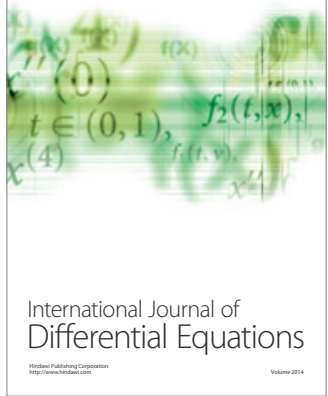
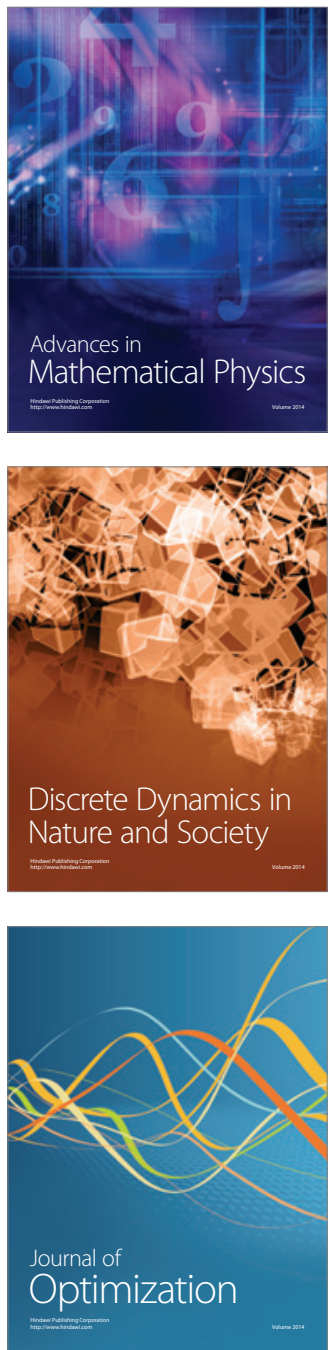